\title{
Calculation of the current noise spectrum in mesoscopic transport: A quantum master equation approach
}

\author{
JunYan Luo, ${ }^{1,2}$ Xin-Qi Li, ${ }^{1,2, *}$ and YiJing Yan ${ }^{2}$ \\ ${ }^{1}$ State Key Laboratory for Superlattices and Microstructures, Institute of Semiconductors, Chinese Academy of Sciences, P.O. Box 912, \\ Beijing 100083, China \\ ${ }^{2}$ Department of Chemistry, Hong Kong University of Science and Technology, Kowloon, Hong Kong, China
}

(Received 10 March 2007; revised manuscript received 23 May 2007; published 16 August 2007)

\begin{abstract}
Based on our recent work on quantum transport [X. Q. Li et al., Phys. Rev. B 71, 205304 (2005)], we show how an efficient calculation can be performed for the current noise spectrum. Compared to the classical rate equation or the quantum trajectory method, the proposed approach is capable of tackling both the many-body Coulomb interaction and quantum coherence on an equal footing. The practical applications are illustrated by transport through quantum dots. We find that this alternative approach is in a certain sense simpler and more straightforward than the well-known Landauer-Büttiker scattering matrix theory.
\end{abstract}

DOI: 10.1103/PhysRevB.76.085325 PACS number(s): 73.23. - b, 73.63.-b, 72.70.+ m, 72.10.Bg

\section{INTRODUCTION}

A modern trend in the study of electric transport in mesoscopic systems concerns not only current-voltage characteristics, but also noise properties. ${ }^{1-13}$ The noise spectrum measures the temporal correlation between individual electron events. It has been proven to be a powerful tool to reveal different possible mechanisms which are not accessible by the mean current measurement. ${ }^{14}$ In particular, via the measurement of current noise one is able to extract information on the system parameters that govern the transport, as well as the internal energy scales of the mesoscopic system.

In principle, a full quantum mechanical calculation for transport noise spectrum can be performed by either the Landauer-Büttiker scattering matrix approach ${ }^{14,15}$ or the nonequilibrium Green's function (NGF) technique. ${ }^{16,17}$ In practice, however, these two approaches have largely been restricted to noninteracting systems. It is noticed that an NGFbased calculation on the zero-frequency noise of transport through a strong Coulomb interaction quantum dot system ${ }^{18}$ and frequency-dependent current noise through quantum-dot spin valve ${ }^{13}$ has been carried out recently with the aid of the advanced real-time diagrammatic technique. ${ }^{19}$

An alternative method to calculate transport noise is the classical rate equation approach. ${ }^{20-24}$ This approach is much simpler than full quantum mechanical methods, thus having been employed in some interesting transport systems-for instance, Coulomb blockade systems. ${ }^{25,26}$ Nevertheless, owing to the classical nature of this approach, quantum coherence is out of the scope of its description. Focused on the effect of quantum coherence in the noise spectrum, Sun and Milburn studied transport through a pair of coupled quantum dots. ${ }^{27}$ For this specific system, they derived a Lindblad-type master equation, then unraveled it to calculate the noise spectrum. It is worth mentioning here that the $n$-resolved quantum Bloch equation approach had been proposed sometime earlier by Gurvitz and Prager ${ }^{28}$ for the same purpose. Here $n$ stands for the electron number tunneled through the junctions. Recently, this approach was elegantly applied to quantum transport problems involving such as quantum interference phenomena ${ }^{29,30}$ and the quantum shuttle mechanism. ${ }^{31,32}$ However, as it starts with the many-particle Schrödinger equation of the entire system, this approach is by far restricted to zero temperature, and also the large bias voltage regime, due to the approximation involved there. We have recently extended the $n$-resolved approach to finitetemperature and finite-voltage cases. ${ }^{33-36}$ The resulting $n$-resolved quantum master equation can serve as a convenient starting point for both the current and measurement noise in a general transport system.

In this work, we complete the master-equation-based transport formalism by developing an efficient method for noise spectrum calculation. After a brief review of the $n$-resolved transport master equation, ${ }^{33-36}$ we present in Sec. II compact formulas for the transport current and noise spectrum. In Sec. III we demonstrate the formalism by the transport through a noninteracting and an interacting quantum dot. For the former, we readily recover the known result of Chen and Ting, ${ }^{16}$ which was obtained originally via a nonequilibrium path integral treatment of full quantum mechanics. For the latter, we extend the result of zero-frequency noise $^{18}$ to the finite-frequency regime, followed by a brief discussion of the noise characteristics. In Sec. IV we calculate the noise spectrum for transport through a pair of coherently coupled quantum dots. It is found that the current noise is a sensitive probe of both intradot and interdot interactions. Finally, concluding remarks are given in Sec. V.

\section{FORMALISM}

\section{A. Conditional quantum master equation}

Consider the typical transport setup as schematically shown in Fig. 1. It consists of the central transport system (device) and two biased electrodes. The total system Hamiltonian reads

$$
\begin{aligned}
H= & H_{\mathrm{S}}\left(c_{\mu}^{\dagger}, c_{\mu}\right)+\sum_{\alpha=\mathrm{L}, \mathrm{R}} \sum_{k} E_{\alpha k} a_{\alpha k}^{\dagger} a_{\alpha k}+\sum_{\alpha=\mathrm{L}, \mathrm{R}} \sum_{k, \mu}\left(t_{\alpha k \mu} c_{\mu}^{\dagger} a_{\alpha k}\right. \\
& + \text { H.c. }) .
\end{aligned}
$$

$H_{\mathrm{S}}$ is the central system Hamiltonian, including possible many-body interactions, and $c_{\mu}^{\dagger}\left(c_{\mu}\right)$ the electron creation 


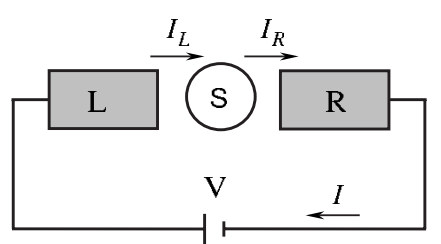

FIG. 1. Schematic setup for electron transport through a mesoscopic system.

(annihilation) operator of state $\mu$, which labels here both the orbital and spin states. The second term describes the noninteracting electrons of the two electrodes. The last term describes tunneling between the central system and the electrodes. Throughout this paper, we assume a unit system of $\hbar=e=1$.

For convenience, we reexpress the tunneling Hamiltonian as

$$
H^{\prime}=\sum_{\mu}\left(c_{\mu}^{\dagger} F_{\mu}+\text { H.c. }\right),
$$

where $F_{\mu}=F_{\mathrm{L} \mu}+F_{\mathrm{R} \mu} \equiv \Sigma_{\alpha=\mathrm{L}, \mathrm{R}} \Sigma_{k} t_{\alpha k \mu} a_{\alpha \mu}$. By treating the tunneling Hamiltonian as perturbation, the second-order cumulant expansion leads to the master equation for the reduced density matrix of the central transport system: ${ }^{37}$

$$
\dot{\rho}(t)=-i \mathcal{L} \rho(t)-\int_{0}^{\infty} d \tau\left\langle\mathcal{L}^{\prime}(t) \mathcal{G}(t, \tau) \mathcal{L}^{\prime}(\tau) \mathcal{G}^{\dagger}(t, \tau)\right\rangle \rho(t) .
$$

Here the reduced density matrix $\rho(t)$ is introduced by tracing over the electrode states from the density matrix $\rho_{\mathrm{T}}$ of the entire system-i.e., $\rho(t) \equiv \operatorname{Tr}_{\mathrm{B}}\left[\rho_{\mathrm{T}}(t)\right]$ and $\langle\cdots\rangle \equiv \operatorname{Tr}_{\mathrm{B}}\left[(\cdots) \rho_{\mathrm{B}}\right]$, with $\rho_{\mathrm{B}}$ the density operator of the electrodes. The Liouvillian superoperators are defined as $\mathcal{L} A \equiv\left[H_{\mathrm{S}}, A\right], \quad \mathcal{L}^{\prime} A$ $\equiv\left[H^{\prime}, A\right]$, and $\mathcal{G}(t, \tau) A \equiv G(t, \tau) A G^{\dagger}(t, \tau)$, where $G(t, \tau)$ is the usual propagator (Hilbert-space Green's function) associated with the system Hamiltonian $H_{\mathrm{S}}$ in the absence of electrode bath.

The trace in Eq. (3) is over all electrode degrees of freedom, leading thus to the equation of motion for the unconditional reduced density matrix of the system. To describe the transport problem, we keep track of the number of electrons that have tunneled through the right (left) junction. To this end, the average over the reservoir states in the entire Hilbert space "B" in Eq. (3) should be replaced with states in the subspace " $\mathrm{B}^{(n)}$," which corresponds to the event of $n$ electrons tunneled through the right or left junction. Let us take the right junction as an example. The trace of Eq. (3) over $\mathrm{B}^{\left(n_{\mathrm{R}}\right)}$ leads to a master-type equation for the conditional reduced state ${ }^{36}$.

$$
\begin{aligned}
\dot{\rho}^{\left(n_{\mathrm{R}}\right)}= & -i \mathcal{L} \rho^{\left(n_{\mathrm{R}}\right)}-\frac{1}{2} \sum_{\mu}\left\{\left[c_{\mu}^{\dagger} A_{\mu}^{(-)} \rho^{\left(n_{\mathrm{R}}\right)}+\rho^{\left(n_{\mathrm{R}}\right)} A_{\mu}^{(+)} c_{\mu}^{\dagger}\right.\right. \\
& -A_{\mathrm{L} \mu}^{(-)} \rho^{\left(n_{\mathrm{R}}\right)} c_{\mu}^{\dagger}-c_{\mu}^{\dagger} \rho^{\left(n_{\mathrm{R}}\right)} A_{\mathrm{L} \mu}^{(+)}-A_{\mathrm{R} \mu}^{(-)} \rho^{\left(n_{\mathrm{R}}-1\right)} c_{\mu}^{\dagger} \\
& \left.\left.-c_{\mu}^{\dagger} \rho^{\left(n_{\mathrm{R}}+1\right)} A_{\mathrm{R} \mu}^{(+)}\right]+ \text {H.c. }\right\} .
\end{aligned}
$$

Here $A_{\alpha \mu}^{(+)}=\Sigma_{\nu} C_{\alpha \nu \mu}^{(+)}(\mathcal{L}) c_{\nu}, \quad A_{\alpha \mu}^{(-)}=\Sigma_{\nu} C_{\alpha \mu \nu}^{(-)}(-\mathcal{L}) c_{\nu}, \quad$ and $\quad A_{\mu}^{( \pm)}$
$=\Sigma_{\alpha} A_{\alpha \mu}^{( \pm)}$. The spectral functions involved, $C_{\alpha \mu \nu}^{( \pm)}( \pm \mathcal{L})$, are defined as

$$
C_{\alpha \mu \nu}^{( \pm)}( \pm \mathcal{L})=2 \int_{0}^{\infty} d t C_{\alpha \mu \nu}^{( \pm)}(t) e^{ \pm i \mathcal{L} t} \approx \int_{-\infty}^{\infty} d t C_{\alpha \mu \nu}^{( \pm)}(t) e^{ \pm i \mathcal{L} t}
$$

with $\quad C_{\alpha \mu \nu}^{(+)}(t-\tau)=\left\langle F_{\alpha \mu}^{\dagger}(t) F_{\alpha \nu}(\tau)\right\rangle \quad$ and $\quad C_{\alpha \mu \nu}^{(-)}(t-\tau)$ $=\left\langle F_{\alpha \mu}(t) F_{\alpha \nu}^{\dagger}(\tau)\right\rangle$, where $\langle\cdots\rangle$ stands for $\operatorname{Tr}_{\mathrm{B}}\left[(\cdots) \rho_{\mathrm{B}}\right]$, the usual meaning of thermal bath average. ${ }^{38}$ The implication of the approximations involved in Eq. (4) and the second identity of Eq. (5) will be elaborated soon.

For $\rho^{\left(n_{\mathrm{L}}\right)}(t)$, which describes the reduced system state conditioned by the electron numbers tunneled through the left junction, a similar equation as Eq. (4) can be obtained by interchanging the indices " $L$ " and "R." With knowledge of the above conditional state $\rho^{\left(n_{\mathrm{L} / \mathrm{R}}\right)}(t)$, we are ready to calculate all the transport properties, such as the transport current and the noise spectrum.

In writing Eqs. (4) and (5), we have employed the standard Born approximation, in which the tunneling Hamiltonian is treated as a perturbation. We have also neglected the dynamical dispersion, as implied in the second identity of Eq. (5), for the sake of later construction of analytical results for the simple but illustrative systems treated in this work. Thus, the system Hamiltonian is a renormalized one, with the static dispersion effect being included. ${ }^{39}$ It is noticed that, in some cases, the dynamic dispersion may lead to some interesting phenomena, such as the negative differential conductance in coupled quantum dots system ${ }^{40}$ and the exchange magnetic field that leads to spin procession. ${ }^{13}$

\section{B. Transport current}

Straightforwardly, the current through the junction $\alpha=\mathrm{L}$ or $\mathrm{R}$ can be calculated via $I_{\alpha}(t)=d\left\langle N_{\alpha}(t)\right\rangle / d t$ $=\sum_{n_{\alpha}} n_{\alpha} \operatorname{Tr}\left[\dot{\rho}^{\left(n_{\alpha}\right)}(t)\right]$, where $\operatorname{Tr}(\cdots)$ means trace over the internal states of the central system. $\operatorname{Tr}\left[\rho^{\left(n_{\alpha}\right)}(t)\right] \equiv P\left(n_{\alpha}, t\right)$ is nothing but the probability by that there are $n_{\alpha}$ electrons tunneled through the junction $\alpha$ until time $t$. Simple algebra leads to ${ }^{36}$

$$
I_{\alpha}(t)=\operatorname{Tr}\left[\mathcal{T}_{\alpha}^{(-)} \rho(t)\right],
$$

with

$$
\mathcal{T}_{\alpha}^{( \pm)}(\cdots)=\frac{1}{2} \sum_{\mu}\left[A_{\alpha \mu}^{(-)}(\cdots) c_{\mu}^{\dagger} \pm c_{\mu}^{\dagger}(\cdots) A_{\alpha \mu}^{(+)}\right]+\text {H.c. }
$$

Here, the unconditional density matrix $\rho(t)$, which is the sum of all $\rho^{\left(n_{\alpha}\right)}(t)$, satisfies a simple unconditional master equation

$$
\begin{gathered}
\dot{\rho}(t)=-i \mathcal{L} \rho(t)-\mathcal{R} \rho(t) \\
\mathcal{R} \rho(t)=\frac{1}{2} \sum_{\mu}\left[c_{\mu}^{\dagger}, A_{\mu}^{(-)} \rho(t)-\rho(t) A_{\mu}^{(+)}\right]+\text {H.c. }
\end{gathered}
$$

\section{Current noise spectrum}

Physically, the current noise spectrum characterizes the temporal fluctuation of the entire circuit current. In steady 
state, the average currents through the left and right junctions are equal to each other, but the temporal (i.e., timedependent) fluctuating currents are not. In general, the circuit current, which is typically the measured quantity in most experiments, is a superposition of the left and right fluctuating currents-i.e., $I(t)=a I_{\mathrm{L}}(t)+b I_{\mathrm{R}}(t)$. Here the coefficients $a$ and $b$ depend on the symmetry of the transport setup (e.g., the junction capacitances) (Ref. 14) and satisfy $a+b=1$. Together with the charge conservation law of $I_{\mathrm{L}}=I_{\mathrm{R}}+\dot{Q}$, where $Q$ denotes the charge in the central system, we obtain

$$
I(t) I(0)=a I_{\mathrm{L}}(t) I_{\mathrm{L}}(0)+b I_{\mathrm{R}}(t) I_{\mathrm{R}}(0)-a b \dot{Q}(t) \dot{Q}(0) .
$$

Accordingly, the noise spectrum is a sum of three parts ${ }^{41,42}$ :

$$
S(\omega)=a S_{\mathrm{L}}(\omega)+b S_{\mathrm{R}}(\omega)-a b S_{c}(\omega) .
$$

Here $S_{\mathrm{L} / \mathrm{R}}(\omega)$ is the noise spectrum of the current through the left (right) junction, while $S_{c}(\omega)$ characterizes the current fluctuations in the central system. In the following we first develop the general formulations for their calculations and then demonstrate them with some representative examples.

For $S_{\mathrm{L} / \mathrm{R}}(\omega)$, we employ the MacDonald's formula ${ }^{43}$

$$
S_{\alpha}(\omega)=2 \omega \int_{0}^{\infty} d t \sin (\omega t) \frac{d}{d t}\left[\left\langle n_{\alpha}^{2}(t)\right\rangle-(\bar{I} t)^{2}\right],
$$

where $\bar{I}$ is the stationary current and $\left\langle n_{\alpha}^{2}(t)\right\rangle=\sum_{n_{\alpha}} n_{\alpha}^{2}\left[\operatorname{Tr} \rho^{\left(n_{\alpha}\right)}(t)\right]$ $=\Sigma_{n} n_{\alpha}^{2} P\left(n_{\alpha}, t\right)$. With the help of Eq. (4), we can show that [cf. Eq. (6b)]

$$
\frac{d}{d t}\left\langle n_{\alpha}^{2}(t)\right\rangle=\operatorname{Tr}\left[2 \mathcal{T}_{\alpha}^{(-)} N^{\alpha}(t)+\mathcal{T}_{\alpha}^{(+)} \rho^{s t}\right],
$$

in which $N^{\alpha}(t) \equiv \sum_{n_{\alpha}} n_{\alpha} \rho^{\left(n_{\alpha}\right)}(t)$ denotes the "particle-number" matrix and $\rho^{\text {st }}$ the stationary density matrix for the calculation of the noise spectrum of steady state. The final expression for $S_{\mathrm{L} / \mathrm{R}}(\omega)$ reads

$$
S_{\alpha}(\omega)=4 \omega \operatorname{Im}\left\{\operatorname{Tr}\left[\mathcal{T}_{\alpha}^{(-)} \tilde{N}^{\alpha}(\omega)\right]\right\}+2 \operatorname{Tr}\left[\mathcal{T}_{\alpha}^{(+)} \rho^{\mathrm{st}}\right]-8 \pi \vec{I}^{2} \delta(\omega),
$$

where $\tilde{N}^{\alpha}(\omega)=\int_{0}^{\infty} d t N^{\alpha}(t) e^{(i \omega-\varepsilon) t}$ with $\varepsilon \rightarrow 0^{+}$introduced for convergence. The last term in Eq. (12) originates from the second term in the MacDonald's formula, Eq. (10). For $\tilde{N}^{\alpha}(\omega)$, we establish the equation of motion for its timedomain counterpart; i.e.,

$$
\frac{d}{d t} N^{\alpha}(t)=-i \mathcal{L} N^{\alpha}(t)-\mathcal{R} N^{\alpha}(t)+\mathcal{T}_{\alpha}^{(-)} \rho^{\mathrm{st}},
$$

whose resolution reads formally as

$$
-(i \omega-\varepsilon) \tilde{N}^{\alpha}(\omega)=-i \mathcal{L} \tilde{N}^{\alpha}(\omega)-\mathcal{R} \tilde{N}^{\alpha}(\omega)+\mathcal{T}_{\alpha}^{(-)} \tilde{\rho}(\omega),
$$

with $\tilde{\rho}(\omega)=i \rho^{\text {st }} /(\omega+i \varepsilon)$ being used here. It results in a $\delta$-function divergence when the solution of Eq. (14) is substituted into Eq. (12). This divergence is canceled by the $\delta$-function term in the second line of Eq. (12). Finally, the noise spectrum $S_{\alpha}(\omega)$ remains regular also in the $\omega \rightarrow 0$ limit.
For the charge fluctuations on the central system, the symmetrized noise spectrum reads

$$
S_{c}(\omega)=\omega^{2} \int_{-\infty}^{\infty} d \tau\langle N(\tau) N+N N(\tau)\rangle e^{i \omega \tau},
$$

where $\langle N(\tau) N\rangle=\operatorname{Tr} \operatorname{Tr}_{\mathrm{B}}\left[U^{\dagger}(\tau) N U(\tau) N \rho^{\mathrm{st}} \rho_{\mathrm{B}}\right]$, with $U(\tau)$ $=e^{-i H \tau}$ and $N$ the electron-number operator of the central system. Using the cyclic property under trace, we have $\langle N(\tau) N\rangle=\operatorname{Tr}[N \sigma(\tau)]$, where $\sigma(\tau)=\operatorname{Tr}_{\mathrm{B}}\left[U(\tau) N \rho^{\text {st }} \rho_{\mathrm{B}} U^{\dagger}(\tau)\right]$ is introduced within the standard Born approximation. Noticeably, $\sigma(\tau)$ is nothing but an alternative reduced matrix, satisfying the same equation (7) of the usual reduced density matrix $\rho(\tau)$, but with the initial condition $\sigma(0)=N \rho^{\text {st }}$. Its Fourier transform $\widetilde{\sigma}(\omega)$ can be easily solved from

$$
i(\omega-\mathcal{L}) \widetilde{\sigma}(\omega)=\mathcal{R} \widetilde{\sigma}(\omega)-N \rho^{\mathrm{st}} .
$$

Then, $S_{c}(\omega)=2 \omega^{2} \operatorname{Re}\{\operatorname{Tr}\{N[\widetilde{\sigma}(\omega)+\widetilde{\sigma}(-\omega)]\}$. More specifically, in most cases we can carry out this quantity in the eigenstate basis of $N$-that is,

$$
S_{c}(\omega)=2 \omega^{2} \operatorname{Re}\left\{\sum_{k} N_{k}\left[\widetilde{\sigma}_{k}(\omega)+\widetilde{\sigma}_{k}(-\omega)\right]\right\},
$$

where $N_{k}$ is the eigenvalue of $N$ for the eigenstate $|k\rangle$, and $\widetilde{\sigma}_{k}(\omega)=\langle k|\widetilde{\sigma}(\omega)| k\rangle$.

We have now constructed a general and quite compact formulation for quantum transport through mesoscopic systems, particularly for the calculation of the noise spectrum which is usually a difficult problem by other approaches. The advantages of this formulation include its straightforwardness, simplicity, and applicability to handle many-electron correlation, quantum coherence, and possible inelastic scattering (dissipative) processes during transport on equal footing.

\section{TRANSPORT THROUGH A SINGLE QUANTUM DOT}

\section{A. Noninteracting quantum dot}

We are in the position to demonstrate the above formalism by transport through a noninteracting quantum dot and then an interacting one. Consider in this subsection the simplest noninteracting case that only a single dot state is involved in the transport process. We ignore the Coulomb electronelectron interaction inside the dot and the spin degrees of freedom. With the standard simplifications, the dot Hamiltonian reads $H_{S}=E_{0} N$, where $N=c^{\dagger} c$ is the number operator.

To proceed, we carry out the electron reservoir (i.e., the electrode) correlation function. Explicitly, $C_{\alpha}^{( \pm)}(t-\tau)$ $=\left|t_{\alpha}\right|^{2} \sum_{k} e^{ \pm i E_{k}(t-\tau)} f_{\alpha}^{( \pm)}\left(E_{k}\right)$, and its Fourier transform $C_{\alpha}^{( \pm)}( \pm \mathcal{L})$ $=\Gamma_{\alpha} f_{\alpha}^{( \pm)}(-\mathcal{L})$. Here, the wideband approximation for the electrodes is applied, resulting in the energy-independent coupling constant $\Gamma_{\alpha}=2 \pi g_{\alpha}\left|t_{\alpha}\right|^{2}$, where $g_{\alpha}$ is the density of states, $f_{\alpha}^{(+)}$is the usual Fermi function, and $f_{\alpha}^{(-)} \equiv 1-f_{\alpha}$. Since $\mathcal{L}^{n} c=\left(-E_{0}\right)^{n} c$, we have $A_{\alpha}^{( \pm)}=C_{\alpha}^{( \pm)}( \pm \mathcal{L}) c=\Gamma_{\alpha} f_{\alpha}^{( \pm)}\left(E_{0}\right) c$. Neglecting the spin degree of freedom, only two states are involved-i.e., $|0\rangle$ and $|1\rangle$ for the empty and the occupied states, respectively. At large bias, the dot level is away from 
the Fermi edges of the leads, the Fermi functions can be approximated by either one or zero, then the transport master equation (7) simply reads

$$
\begin{aligned}
& \dot{\rho}_{0}=-\Gamma_{\mathrm{L}} \rho_{0}+\Gamma_{\mathrm{R}} \rho_{1}, \\
& \dot{\rho}_{1}=-\Gamma_{\mathrm{R}} \rho_{1}+\Gamma_{\mathrm{L}} \rho_{0} .
\end{aligned}
$$

By inserting the stationary solution of Eq. (18) into the current formula, Eq. (6), the well-known resonant current is obtained: $\bar{I}=\bar{I}_{\mathrm{R}}=-\bar{I}_{\mathrm{L}}=\Gamma_{\mathrm{L}} \Gamma_{\mathrm{R}} / \Gamma$, with $\Gamma=\Gamma_{\mathrm{L}}+\Gamma_{\mathrm{R}}$ the total tunneling width.

Now we turn to the noise spectrum. The current fluctuation spectra of the left and right electrodes following Eq. (12) are

$$
\begin{aligned}
& S_{\mathrm{L}}(\omega)=-4 \omega \Gamma_{\mathrm{L}} \operatorname{Im}\left[\tilde{N}_{0}^{\mathrm{L}}(\omega)\right]+2 \Gamma_{\mathrm{L}} \rho_{0}^{\mathrm{st}}-8 \pi \vec{I}^{2} \delta(\omega), \\
& S_{\mathrm{R}}(\omega)=4 \omega \Gamma_{\mathrm{R}} \operatorname{Im}\left[\tilde{N}_{1}^{\mathrm{R}}(\omega)\right]+2 \Gamma_{\mathrm{R}} \rho_{1}^{\mathrm{st}}-8 \pi \vec{I}^{2} \delta(\omega) .
\end{aligned}
$$

Here, $\rho_{0}^{\text {st }}$ and $\rho_{1}^{\text {st }}$ are the stationary solutions of master equation (18); $\tilde{N}_{0}^{\mathrm{L}}(\omega)$ and $\tilde{N}_{1}^{\mathrm{R}}(\omega)$ can be evaluated based on Eq. (14). For instance, $\tilde{N}_{0}^{\mathrm{L}}(\omega)$ is given by

$$
\begin{gathered}
(i \omega-\varepsilon) \tilde{N}_{0}^{\mathrm{L}}(\omega)=\Gamma_{\mathrm{L}} \tilde{N}_{0}^{\mathrm{L}}(\omega)-\Gamma_{\mathrm{R}} \tilde{N}_{1}^{\mathrm{L}}(\omega), \\
(i \omega-\varepsilon) \widetilde{N}_{1}^{\mathrm{L}}(\omega)=\Gamma_{\mathrm{R}} \widetilde{N}_{1}^{\mathrm{L}}(\omega)-\Gamma_{\mathrm{L}} \tilde{N}_{0}^{\mathrm{L}}(\omega)+\Gamma_{\mathrm{L}} \widetilde{\rho}_{0}(\omega),
\end{gathered}
$$

where $\widetilde{\rho}_{0}(\omega)=i \rho_{0}^{\mathrm{st}} /(\omega+i \varepsilon) . \widetilde{N}^{\mathrm{R}}(\omega)$ can be obtained similarly. Then, straightforwardly, we have

$$
S_{\mathrm{L}}(\omega)=S_{\mathrm{R}}(\omega)=2 \bar{I}\left[\frac{\Gamma_{\mathrm{L}}^{2}+\Gamma_{\mathrm{R}}^{2}}{\Gamma^{2}}+\frac{2 \Gamma_{\mathrm{L}} \Gamma_{\mathrm{R}}}{\Gamma^{2}} \frac{\omega^{2}}{\Gamma^{2}+\omega^{2}}\right] .
$$

Note that the noise spectra of the two tunneling currents are identical for this simple system.

To calculate the charge fluctuation spectrum on the central quantum dot based on Eq. (17), we simply have $S_{c}(\omega)$ $=2 \omega^{2} \operatorname{Re}\left[\widetilde{\sigma}_{1}(\omega)+\widetilde{\sigma}_{1}(-\omega)\right]$, where the fact that $N_{0}=0$ and $N_{1}$ $=1$ have been taken into account. Following Eq. $(16), \widetilde{\sigma}_{1}(\omega)$ can be determined via

$$
\begin{gathered}
i \omega \widetilde{\sigma}_{0}(\omega)=\Gamma_{\mathrm{L}} \widetilde{\sigma}_{0}(\omega)-\Gamma_{\mathrm{R}} \widetilde{\sigma}_{1}(\omega), \\
i \omega \widetilde{\sigma}_{1}(\omega)=\Gamma_{\mathrm{R}} \widetilde{\sigma}_{1}(\omega)-\Gamma_{\mathrm{L}} \widetilde{\sigma}_{0}(\omega)-\rho_{1}^{\mathrm{st}} .
\end{gathered}
$$

Accordingly, we obtain $S_{c}(\omega)=4 \bar{I} \omega^{2} /\left(\Gamma^{2}+\omega^{2}\right)$.

Combining all three components above of the noise spectrum according to Eq. (9) and assuming a symmetric configuration (i.e., $a=b=1 / 2$ ), we obtain

$$
S(\omega)=\bar{I}\left[1+\left(1-\frac{4 \Gamma_{\mathrm{L}} \Gamma_{\mathrm{R}}}{\Gamma^{2}}\right) \frac{\Gamma^{2}}{\Gamma^{2}+\omega^{2}}\right] .
$$

This is the well-known result for resonant tunneling through symmetric double-barrier structures. It was first derived by
Chen and Ting based on the nonequilibrium path-integral technique. ${ }^{16}$ Later it was also obtained by Blanter and Büttiker via the scattering approach, ${ }^{14}$ where the so-called Fano factor $F(\omega)=S(\omega) / 2 \bar{I}$, especially its value at zero frequency $F(0)=1-2 \Gamma_{\mathrm{L}} \Gamma_{\mathrm{R}} / \Gamma^{2}$, was discussed in particular. Here, we have presented an alternative derivation which seems elegant and interesting. More importantly, its generalization to more complicated systems is straightforward, as to be illustrated soon.

\section{B. Interacting quantum dot with Zeeman splitting}

We now consider transport through an interacting quantum dot described by

$$
H_{\mathrm{S}}=\sum_{\sigma} E_{\sigma} N_{\sigma}+U N_{\uparrow} N_{\downarrow},
$$

where $E_{\downarrow}$ and $E_{\uparrow}$ are the electron levels with finite Zeeman splitting and $N_{\sigma}=c_{\sigma}^{\dagger} c_{\sigma}$ is the electron number operator for spin $\sigma$ [it should not be confused with the reduced operator $\sigma(\tau)$ introduced in the calculation of $\left.S_{c}(\omega)\right]$. In this model, the $U$ term accounts for the many-body Coulomb correlation. As in the previous example, we should first carry out the spectral function $C_{\alpha \sigma \sigma^{\prime}}^{( \pm)}$and the operator $A_{\alpha \sigma^{*}}^{( \pm)}$. Since the interaction does not flip the electron spin, the spectral function must be diagonal with respect to the spin indices-i.e., $C_{\alpha \sigma \sigma^{\prime}}^{( \pm)}=\delta_{\sigma \sigma^{\prime}} C_{\alpha \sigma \sigma^{\circ}}^{( \pm)}$. Further, under the wideband approximation, we have $A_{\alpha \sigma}^{( \pm)}=\Gamma_{\alpha} f_{\alpha}^{( \pm)}\left(W_{\sigma}\right) c_{\sigma}$, where $W_{\sigma} \equiv E_{\uparrow} \delta_{\uparrow \sigma}$ $+E_{\downarrow} \delta_{\downarrow \sigma}+U\left(n_{\uparrow} \delta_{\downarrow \sigma}+n_{\downarrow} \delta_{\uparrow \sigma}\right)$ uniquely determines four eigenenergy levels of the interacting quantum dot-i.e., $E_{\downarrow}, E_{\uparrow}, E_{\downarrow}$ $+U$ and $E_{\uparrow}+U$. It sets up four regimes for the applied bias voltage: (i) $\mu_{\mathrm{L}}>E_{\downarrow}>\mu_{\mathrm{R}}$, (ii) $\mu_{\mathrm{L}}>E_{\uparrow}, E_{\downarrow}>\mu_{\mathrm{R}}$, (iii) $\mu_{\mathrm{L}}$ $>E_{\downarrow}+U, E_{\uparrow}, E_{\downarrow}>\mu_{\mathrm{R}}$, and (iv) $\mu_{\mathrm{L}}>E_{\uparrow}+U, E_{\downarrow}+U, E_{\uparrow}, E_{\downarrow}$ $>\mu_{\mathrm{R}}$.

For the sake of being compact, here we only present a detailed derivation for regime (ii). In this regime, the involved states include $|0\rangle$ (empty dot), $|\uparrow\rangle$ (occupation by a spin-up electron), and $|\downarrow\rangle$ (occupation by a spin-down electron). Accordingly, the transport master equation reads

$$
\begin{gathered}
\dot{\rho}_{0}=-2 \Gamma_{\mathrm{L}} \rho_{0}+\Gamma_{\mathrm{R}}\left(\rho_{\downarrow}+\rho_{\uparrow}\right), \\
\dot{\rho}_{\downarrow}=-\Gamma_{\mathrm{R}} \rho_{\downarrow}+\Gamma_{\mathrm{L}} \rho_{0}, \\
\dot{\rho}_{\uparrow}=-\Gamma_{\mathrm{R}} \rho_{\uparrow}+\Gamma_{\mathrm{L}} \rho_{0} .
\end{gathered}
$$

Its stationary solution gives rise to the steady-state current $\bar{I}=\bar{I}_{\mathrm{R}}=-\bar{I}_{\mathrm{L}}=2 \Gamma_{\mathrm{L}} \Gamma_{\mathrm{R}} / \Gamma_{\text {eff }}$, with $\Gamma_{\text {eff }}=2 \Gamma_{\mathrm{L}}+\Gamma_{\mathrm{R}}$.

In the aforementioned representation of occupation states, $S_{\mathrm{L} / \mathrm{R}}(\omega)$ are expressed as

$$
S_{\mathrm{L}}(\omega)=-8 \omega \Gamma_{\mathrm{L}} \operatorname{Im}\left[\tilde{N}_{0}^{\mathrm{L}}(\omega)\right]+4 \Gamma_{\mathrm{L}} \rho_{0}^{\mathrm{st}}-8 \pi \vec{I}^{2} \delta(\omega),
$$

$$
\begin{aligned}
S_{\mathrm{R}}(\omega)= & 4 \omega \Gamma_{\mathrm{R}} \operatorname{Im}\left[\tilde{N}_{\uparrow}^{\mathrm{R}}(\omega)+\tilde{N}_{\downarrow}^{\mathrm{R}}(\omega)\right]+2 \Gamma_{\mathrm{R}}\left(\rho_{\uparrow}^{\mathrm{st}}+\rho_{\downarrow}^{\mathrm{st}}\right) \\
& -8 \pi \bar{I}^{2} \delta(\omega) .
\end{aligned}
$$


TABLE I. Zero-frequency and frequency-dependent noise components in the different bias regimes, which are characterized by (i) $\mu_{\mathrm{L}}>E_{\downarrow}>\mu_{\mathrm{R}}$, (ii) $\mu_{\mathrm{L}}>E_{\uparrow}, E_{\downarrow}>\mu_{\mathrm{R}}$, (iii) $\mu_{\mathrm{L}}>E_{\downarrow}+U, E_{\uparrow}, E_{\downarrow}>\mu_{\mathrm{R}}$, and (iv) $\mu_{\mathrm{L}}>E_{\uparrow}+U, E_{\downarrow}+U, E_{\uparrow}, E_{\downarrow}>\mu_{\mathrm{R}}$, respectively. In the results shown in the table, $\Gamma=\Gamma_{\mathrm{L}}+\Gamma_{\mathrm{R}}$ is introduced for the total level-broadening width, $\Gamma_{\mathrm{eff}}=2 \Gamma_{\mathrm{L}}+\Gamma_{\mathrm{R}}$ for effective tunneling width, and $Z=\Gamma^{3}-\Gamma_{\mathrm{L}}^{2} \Gamma_{\mathrm{R}}+\Gamma_{\mathrm{L}} \Gamma_{\mathrm{R}}^{2}+5 \Gamma_{\mathrm{R}}^{3}$ for brevity.

\begin{tabular}{|c|c|c|c|c|}
\hline Bias regime & (i) & (ii) & (iii) & (iv) \\
\hline$S(0)$ & $\frac{2 \Gamma_{\mathrm{L}} \Gamma_{\mathrm{R}}\left(\Gamma_{\mathrm{L}}^{2}+\Gamma_{\mathrm{R}}^{2}\right)}{\Gamma^{3}}$ & $\frac{4 \Gamma_{\mathrm{L}} \Gamma_{\mathrm{R}}\left(4 \Gamma_{\mathrm{L}}^{2}+\Gamma_{\mathrm{R}}^{2}\right)}{\Gamma_{\text {eff }}^{3}}$ & $\frac{2 \Gamma_{L} \Gamma_{R}\left(\Gamma_{L}+2 \Gamma_{R}\right)}{\Gamma^{5}}\left(\Gamma^{3}-3 \Gamma_{L} \Gamma_{R}^{2}\right)$ & $\frac{4 \Gamma_{\mathrm{L}} \Gamma_{\mathrm{R}}\left(\Gamma_{\mathrm{L}}^{2}+\Gamma_{\mathrm{R}}^{2}\right)}{\Gamma^{3}}$ \\
\hline$S_{\mathrm{L}}(\omega)$ & $\frac{4 \Gamma_{\mathrm{L}}^{2} \Gamma_{\mathrm{R}}^{2}}{\Gamma^{3}} \frac{\omega^{2}}{\Gamma^{2}+\omega^{2}}$ & $\frac{16 \Gamma_{L}^{2} \Gamma_{\mathrm{R}}^{2}}{\Gamma_{\text {eff }}^{3}} \frac{\omega^{2}}{\Gamma_{\text {eff }}^{2}+\omega^{2}}$ & $\frac{2 \Gamma_{\mathrm{L}}^{2} \Gamma_{\mathrm{R}} Z \omega^{2}}{\Gamma^{5}\left(\Gamma^{2}+\omega^{2}\right)}+\frac{4 \Gamma_{\mathrm{L}}^{3} \Gamma_{\mathrm{R}}\left(\Gamma_{\mathrm{L}}^{2}-2 \Gamma_{\mathrm{R}}^{2}\right) \omega^{2}}{\Gamma^{3}\left(\Gamma^{2}+\omega^{2}\right)^{2}}$ & $\frac{8 \Gamma_{L}^{2} \Gamma_{\mathrm{R}}^{2}}{\Gamma^{3}} \frac{\omega^{2}}{\Gamma^{2}+\omega^{2}}$ \\
\hline$S_{\mathrm{R}}(\omega)$ & $\frac{4 \Gamma_{\mathrm{L}}^{2} \Gamma_{\mathrm{R}}^{2}}{\Gamma^{3}} \frac{\omega^{2}}{\Gamma^{2}+\omega^{2}}$ & $\frac{16 \Gamma_{L}^{2} \Gamma_{\mathrm{R}}^{2}}{\Gamma_{\mathrm{eff}}^{3}} \frac{\omega^{2}}{\Gamma_{\text {eff }}^{2}+\omega^{2}}$ & $\frac{6 \Gamma_{\mathrm{L}}^{2} \Gamma_{\mathrm{R}}^{3}\left(\Gamma_{\mathrm{L}}+2 \Gamma_{\mathrm{R}}\right) \omega^{2}}{\Gamma^{5}\left(\Gamma^{2}+\omega^{2}\right)}-\frac{4 \Gamma_{\mathrm{L}}^{3} \Gamma_{\mathrm{R}}^{2}\left(2 \Gamma_{\mathrm{L}}+3 \Gamma_{\mathrm{R}}\right) \omega^{2}}{\Gamma^{3}\left(\Gamma^{2}+\omega^{2}\right)^{2}}$ & $\frac{8 \Gamma_{\mathrm{L}}^{2} \Gamma_{\mathrm{R}}^{2}}{\Gamma^{3}} \frac{\omega^{2}}{\Gamma^{2}+\omega^{2}}$ \\
\hline$S_{c}(\omega)$ & $\frac{4 \Gamma_{\mathrm{L}} \Gamma_{\mathrm{R}}}{\Gamma} \frac{\omega^{2}}{\Gamma^{2}+\omega^{2}}$ & $\frac{8 \Gamma_{L} \Gamma_{\mathrm{R}}}{\Gamma_{\text {eff }}} \frac{\omega^{2}}{\Gamma_{\text {eff }}^{2}+\omega^{2}}$ & $\frac{2 \Gamma_{\mathrm{L}} \Gamma_{\mathrm{R}}\left(\Gamma^{2}+3 \Gamma_{\mathrm{R}}^{2}\right) \omega^{2}}{\Gamma^{3}\left(\Gamma^{2}+\omega^{2}\right)}+\frac{4 \Gamma_{\mathrm{L}}^{2} \Gamma_{\mathrm{R}}\left(\Gamma_{\mathrm{L}}+2 \Gamma_{\mathrm{R}}\right) \omega^{4}}{\Gamma^{3}\left(\Gamma^{2}+\omega^{2}\right)^{2}}$ & $\frac{8 \Gamma_{\mathrm{L}} \Gamma_{\mathrm{R}}}{\Gamma} \frac{\omega^{2}}{\Gamma^{2}+\omega^{2}}$ \\
\hline
\end{tabular}

$\widetilde{N}_{0}^{\mathrm{L}}(\omega), \tilde{N}_{\uparrow}^{\mathrm{R}}(\omega)$, and $\tilde{N}_{\downarrow}^{\mathrm{R}}(\omega)$ can be obtained from Eq. (14). For $\tilde{N}_{0}^{\mathrm{L}}(\omega)$, for instance, the relevant equations explicitly read

$$
\begin{aligned}
& (i \omega-\varepsilon) \tilde{N}_{0}^{\mathrm{L}}(\omega)=2 \Gamma_{\mathrm{L}} \tilde{N}_{0}^{\mathrm{L}}(\omega)-\Gamma_{\mathrm{R}} \tilde{N}_{\uparrow}^{\mathrm{L}}(\omega)-\Gamma_{\mathrm{R}} \tilde{N}_{\downarrow}^{\mathrm{L}}(\omega), \\
& (i \omega-\varepsilon) \tilde{N}_{\uparrow}^{\mathrm{L}}(\omega)=\Gamma_{\mathrm{R}} \tilde{N}_{\uparrow}^{\mathrm{L}}(\omega)-\Gamma_{\mathrm{L}} \tilde{N}_{0}^{\mathrm{L}}(\omega)+\Gamma_{\mathrm{L}} \tilde{\rho}_{0}(\omega), \\
& (i \omega-\varepsilon) \tilde{N}_{\downarrow}^{\mathrm{L}}(\omega)=\Gamma_{\mathrm{R}} \tilde{N}_{\downarrow}^{\mathrm{L}}(\omega)-\Gamma_{\mathrm{L}} \tilde{N}_{0}^{\mathrm{L}}(\omega)+\Gamma_{\mathrm{L}} \widetilde{\rho}_{0}(\omega) .
\end{aligned}
$$

Note that in the above equations $\widetilde{\rho}(\omega)=i \rho^{\mathrm{st}} /(\omega+i \varepsilon)$ and $\rho^{\mathrm{st}}$ is the stationary solution of Eq. (25). Straightforwardly we obtain

$$
S_{\mathrm{L}}(\omega)=S_{\mathrm{R}}(\omega)=2 \bar{I}\left[\frac{\left(2 \Gamma_{\mathrm{L}}\right)^{2}+\Gamma_{\mathrm{R}}^{2}}{\Gamma_{\mathrm{eff}}^{2}}+\frac{2\left(2 \Gamma_{\mathrm{L}}\right) \Gamma_{\mathrm{R}}}{\Gamma_{\mathrm{eff}}^{2}} \frac{\omega^{2}}{\Gamma_{\mathrm{eff}}^{2}+\omega^{2}}\right]
$$

Also, in the same occupation-state representation, $S_{c}(\omega)$ simply reads $S_{c}(\omega)=2 \omega^{2} \operatorname{Re}\left[\widetilde{\sigma}_{\uparrow}(\omega)+\widetilde{\sigma}_{\downarrow}(\omega)+\widetilde{\sigma}_{\uparrow}(-\omega)+\widetilde{\sigma}_{\downarrow}(-\omega)\right]$, where $\widetilde{\sigma}_{\uparrow}(\omega)$ and $\widetilde{\sigma}_{\downarrow}(\omega)$ can be solved from

$$
\begin{gathered}
i \omega \widetilde{\sigma}_{0}(\omega)=2 \Gamma_{\mathrm{L}} \widetilde{\sigma}_{0}(\omega)-\Gamma_{\mathrm{R}} \widetilde{\sigma}_{\uparrow}(\omega)-\Gamma_{\mathrm{R}} \widetilde{\sigma}_{\downarrow}(\omega), \\
i \omega \widetilde{\sigma}_{\uparrow}(\omega)=\Gamma_{\mathrm{R}} \widetilde{\sigma}_{\uparrow}(\omega)-\Gamma_{\mathrm{L}} \widetilde{\sigma}_{0}(\omega)-\rho_{\uparrow}^{\mathrm{st}}, \\
i \omega \widetilde{\sigma}_{\downarrow}(\omega)=\Gamma_{\mathrm{R}} \widetilde{\sigma}_{\downarrow}(\omega)-\Gamma_{\mathrm{L}} \widetilde{\sigma}_{0}(\omega)-\rho_{\downarrow}^{\mathrm{st}} .
\end{gathered}
$$

Its solution gives

$$
S_{c}(\omega)=4 \bar{I}\left[\frac{\omega^{2}}{\Gamma_{\mathrm{eff}}^{2}+\omega^{2}}\right]
$$

Listed in Table I are all the components of the noise spectrum, together with their corresponding voltage regions, for which the derivation is completely the same as above.

In Ref. 18 the zero-frequency noise was carried out for the same system of this example based on a sophisticated nonequilibrium real time diagrammatic technique. Here we have extended the result to the finite-frequency regime using a much simpler and more straightforward approach. We notice that the noise spectra in regimes (i) and (iv) differ from each other only by an overall factor of 2 . The reason is that the electron near the Fermi surface of the left electrode can only pass through the spin-down level of the quantum dot in regime (i), but can freely pass through the two levels in regime (iv) due to complete overcoming the Coulomb blockade. Consequently, the result in regime (iv) is also the same as that of the noninteracting dot obtained in the previous subsection. In the Coulomb blockade regime (ii), electron can only pass through either the spin-down or the spin-up level in an exclusive manner. Compared to the situation of regime (i), the entrance probability of electron from the left electrode to the dot is enhanced by a factor of 2 , whereas the leaving probability to the right electrode is the same. Therefore, in all the components of noise spectrum in regime (ii), $2 \Gamma_{\mathrm{L}}$ replaces the $\Gamma_{\mathrm{L}}$ in the result of regime (i). In regime (iii), the Coulomb blockade is partially overcome; i.e., an electron can enter the dot even if there has been already an electron on the spin-up state in the quantum dot. The noise spectrum in this regime is relatively complicated and seems beyond an intuitively simple interpretation. However, the unique features of noise spectrum in regimes (ii) and (iii) may provide a pathway to distinguish Coulomb blockade phenomena from that resulting from noninteracting multilevels, as emphasized in Ref. 18 based on the zero-frequency noise. Moreover, the "asymmetry" of $\Gamma_{\mathrm{L}}$ and $\Gamma_{\mathrm{R}}$ may provide a useful tool to determine their respective values, which is very important in molecular electronics, due to the need to identify the subtle connection of the molecule with the electrodes.

\section{TRANSPORT THROUGH TWO COUPLED QUANTUM DOTS}

In this section we study the noise characteristics of transport through a pair of coupled quantum dots, as shown in Fig. 2. Under proper voltage conditions, we assume that there is only a single energy level in each dot involved in the transport. Thus the relevant Hamiltonian reads 


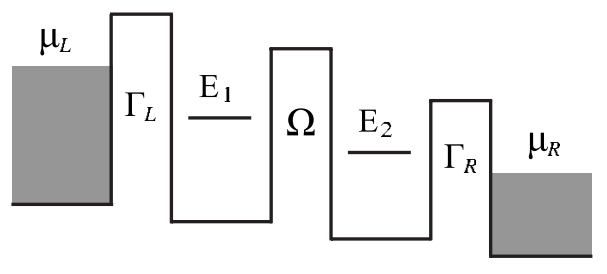

FIG. 2. Schematic illustration for transport through a pair of coupled quantum dots.

$$
\begin{aligned}
\hat{H}_{S}= & \sum_{i=1,2} \sum_{\sigma=\uparrow, \downarrow} E_{i} N_{i \sigma}+U_{0} \sum_{i=1,2} N_{i \uparrow} N_{i \downarrow}+U_{12} N_{1} N_{2} \\
& +\sum_{\sigma=\uparrow, \downarrow} \Omega\left(c_{1 \sigma}^{\dagger} c_{2 \sigma}+c_{2 \sigma}^{\dagger} c_{1 \sigma}\right),
\end{aligned}
$$

where $N_{i \sigma}=c_{i \sigma}^{\dagger} c_{i \sigma}$ is the number operator of electrons with spin $\sigma$ in the $i$ th dot and $N_{i}=\sum_{\sigma} N_{i \sigma}$. The coherent coupling between the two quantum dots and the intradot (interdot) Coulomb interaction are characterized by $\Omega$ and $U_{0}\left(U_{12}\right)$, respectively.

The noise spectrum under a noninteracting limit has been carried out in Ref. 27 based on the quantum trajectory theory. Since typically the quantum trajectory theory is established by unraveling the Lindblad-type master equation, this approach may have certain limitations in practice. For instance, in the presence of many-body Coulomb interactions, the master equation for transport through quantum dots is not in the Lindblad form. In the following we show that the $n$-resolved master equation approach can easily handle this problem. In particular, we assume that the on-site intradot interaction is relatively strong so that $E_{1,2}+U_{0}>\mu_{\mathrm{L}}$ $>\mu_{\mathrm{R}}$. It implies that each dot can be occupied by at most one electron. For the interdot Coulomb interaction, we separately consider two cases: (i) $\mu_{\mathrm{L}}>E_{1,2}+U_{12}>\mu_{\mathrm{R}}$ and (ii) $E_{1,2}$ $+U_{12}>\mu_{\mathrm{L}}>\mu_{\mathrm{R}}$

\section{A. Weak interdot interaction}

This corresponds to the condition $\mu_{\mathrm{L}}>E_{1,2}+U_{12}>\mu_{\mathrm{R}}$ and is referred to as the single-dot Coulomb blockade regime since two electrons can occupy the coupled dots. The involved states are $|0\rangle$ for the two dots empty, $|1 \sigma\rangle$ for the first dot occupied, $|2 \sigma\rangle$ for the second dot occupied, and $\left|1 \sigma 2 \sigma^{\prime}\right\rangle$ for both dots occupied. In this state basis, the resultant master equation reads

$$
\begin{gathered}
\dot{\rho}_{0}=-2 \Gamma_{\mathrm{L}} \rho_{0}+\Gamma_{\mathrm{R}}\left[\rho_{2 \uparrow}+\rho_{2 \downarrow}\right], \\
\dot{\rho}_{1 \uparrow}=i \Omega\left[\rho_{2 \uparrow}^{1 \uparrow}-\rho_{1 \uparrow}^{2 \uparrow}\right]+\Gamma_{\mathrm{L}} \rho_{0}+\Gamma_{\mathrm{R}}\left[\rho_{1 \uparrow 2 \uparrow}+\rho_{1 \uparrow 2 \downarrow}\right], \\
\dot{\rho}_{2 \uparrow}=-i \Omega\left[\rho_{2 \uparrow}^{1 \uparrow}-\rho_{1 \uparrow}^{2 \uparrow}\right]-\left(2 \Gamma_{\mathrm{L}}+\Gamma_{\mathrm{R}}\right) \rho_{2 \uparrow}, \\
\dot{\rho}_{1 \uparrow 2 \uparrow}=-\Gamma_{\mathrm{R}} \rho_{1 \uparrow 2 \uparrow}+\Gamma_{\mathrm{L}} \rho_{2 \uparrow}, \\
\dot{\rho}_{2 \uparrow}^{1 \uparrow}=i \Delta \rho_{2 \uparrow}^{1 \uparrow}+i \Omega\left[\rho_{1 \uparrow}-\rho_{2 \uparrow}\right]-\frac{1}{2}\left(2 \Gamma_{\mathrm{L}}+\Gamma_{\mathrm{R}}\right) \rho_{2 \uparrow}^{1 \uparrow},
\end{gathered}
$$

where the nondiagonal matrix element $\rho_{2 \uparrow}^{1 \uparrow}=\langle 1 \uparrow|\rho| 2 \uparrow\rangle$ and the level off-resonance $\Delta=E_{2}-E_{1}$. Here, for brevity, we only show equations for the spin-up components. Similar equations hold also for the spin-down components.

The stationary solution of Eq. (32) straightforwardly leads to the steady-state current as

$$
\bar{I}=\frac{4 \gamma_{1} \Gamma_{\mathrm{L}} \Gamma_{\mathrm{R}} \Omega^{2}}{\Gamma_{\mathrm{L}} \Gamma_{\mathrm{R}}\left(\gamma_{1}^{2}+4 \Delta^{2}\right)+2 \gamma_{1}^{2} \Omega^{2}},
$$

where $\gamma_{1} \equiv 2 \Gamma_{\mathrm{L}}+\Gamma_{\mathrm{R}}$ is introduced. Compared to the "spinless" situation, the possible occupation states of the double dots are the same. However, the entrance probability of electron from the left electrode is enhanced by a factor of 2 , whereas the leaving probability to the right electrode keeps unchanged. This leads to the above result differing from that in Refs. 28 and 44, where either the spin degree of freedom or the Coulomb interaction was not fully taken into account.

To evaluate the current noise spectrum, we first employ MacDonald's formula, Eq. (10). For $S_{\mathrm{L}}(\omega)$, we have

$$
\begin{aligned}
S_{\mathrm{L}}(\omega)= & -8 \omega \Gamma_{\mathrm{L}} \operatorname{Im}\left[\tilde{N}_{0}(\omega)+\tilde{N}_{2 \uparrow}(\omega)+\tilde{N}_{2 \downarrow}(\omega)\right]+4 \Gamma_{\mathrm{L}}\left(\rho_{0}^{\mathrm{st}}\right. \\
& \left.+\rho_{2 \uparrow}^{\mathrm{st}}+\rho_{2 \downarrow}^{\mathrm{st}}\right)-8 \pi \vec{I}^{2} \delta(\omega),
\end{aligned}
$$

where $\rho^{\text {st }}$ is the stationary solution of Eq. (32) and $\tilde{N}(\omega)$ $=\int_{0}^{\infty} d t e^{(i \omega-\varepsilon) t} N(t)$ with $N(t)$ the matrix associated with the number of electrons tunneled through the left junction. $\tilde{N}(\omega)$ can be obtained from the coupled equations

$$
(i \omega-\varepsilon) \tilde{N}_{0}(\omega)=2 \Gamma_{\mathrm{L}} \tilde{N}_{0}(\omega)-\Gamma_{\mathrm{R}}\left[\tilde{N}_{2 \uparrow}(\omega)+\tilde{N}_{2 \downarrow}(\omega)\right],
$$

$$
\begin{aligned}
(i \omega-\varepsilon) \tilde{N}_{1 \uparrow}(\omega)= & -\Gamma_{\mathrm{L}} \tilde{N}_{0}(\omega)-i \Omega\left[\tilde{N}_{2 \uparrow}^{1 \uparrow}(\omega)-\tilde{N}_{1 \uparrow}^{2 \uparrow}(\omega)\right] \\
& -\Gamma_{\mathrm{R}}\left[\tilde{N}_{1 \uparrow 2 \uparrow}(\omega)+\tilde{N}_{1 \uparrow 2 \downarrow}(\omega)\right]+\Gamma_{\mathrm{L}} \tilde{\rho}_{0}(\omega),
\end{aligned}
$$

$$
\begin{aligned}
(i \omega-\varepsilon) \tilde{N}_{2 \uparrow}(\omega)=\left(2 \Gamma_{\mathrm{L}}+\Gamma_{\mathrm{R}}\right) \tilde{N}_{2 \uparrow}(\omega)+i \Omega\left[\tilde{N}_{2 \uparrow}^{1 \uparrow}(\omega)-\tilde{N}_{1 \uparrow}^{2 \uparrow}(\omega)\right], & (35 \mathrm{c}) \\
(i \omega-\varepsilon) \tilde{N}_{1 \uparrow 2 \uparrow}(\omega)= & \Gamma_{\mathrm{R}} \tilde{N}_{1 \uparrow 2 \uparrow}(\omega)-\Gamma_{\mathrm{L}} \tilde{N}_{2 \uparrow}(\omega)+\Gamma_{\mathrm{L}} \tilde{\rho}_{2 \uparrow}(\omega), \\
(i \omega-\varepsilon) \tilde{N}_{2 \uparrow}^{1 \uparrow}(\omega)= & -i \Delta \tilde{N}_{2 \uparrow}^{1 \uparrow}(\omega)-i \Omega\left[\tilde{N}_{1 \uparrow}(\omega)-\tilde{N}_{2 \uparrow}(\omega)\right] \\
& +\frac{1}{2}\left(2 \Gamma_{\mathrm{L}}+\Gamma_{\mathrm{R}}\right) \tilde{N}_{2 \uparrow}^{1 \uparrow}(\omega),
\end{aligned}
$$

where $\tilde{\rho}(\omega)=i \rho^{\mathrm{st}} /(\omega+i \varepsilon)$. Straightforwardly, based on the solution of the above equations, the zero-frequency Fano factor is found to be

$$
\begin{aligned}
F(0) \equiv & S(0) / 2 \bar{I}=1 \\
& -\frac{4 \Gamma_{\mathrm{L}} \Gamma_{\mathrm{R}} \Omega^{2}\left[4 \gamma_{1}^{2}\left(\Delta^{2}+\Omega^{2}\right)+2 \Gamma_{\mathrm{L}} \Gamma_{\mathrm{R}}\left(\gamma_{1}^{2}-12 \Delta^{2}\right)+\gamma_{1}^{4}\right]}{\left[\Gamma_{\mathrm{L}} \Gamma_{\mathrm{R}}\left(\gamma_{1}^{2}+4 \Delta^{2}\right)+2 \gamma_{1}^{2} \Omega^{2}\right]^{2}} .
\end{aligned}
$$


For the finite-frequency noise spectrum, the numerical result is shown in Fig. 3(a) by the dashed curve, where the adopted parameters are $\Gamma_{\mathrm{L}}=\Gamma_{\mathrm{R}}=\Gamma, \Delta=0$, and $\Omega=6 \Gamma$. The same result for the noise component $S_{\mathrm{R}}(\omega)$ can be carried out precisely by the same procedures.

Based on the formalism developed in Sec. II, the spectrum of charge-number fluctuations on the double dots reads $\quad S_{c}(\omega)=4 \omega^{2} \operatorname{Re}\left[\widetilde{\sigma}_{1 \uparrow}(\omega)+\widetilde{\sigma}_{2 \uparrow}(\omega)+4 \widetilde{\sigma}_{1 \uparrow 2 \uparrow}(\omega)+\widetilde{\sigma}_{1 \uparrow}(-\omega)\right.$ $\left.+\widetilde{\sigma}_{2 \uparrow}(-\omega)+4 \widetilde{\sigma}_{1 \uparrow 2 \uparrow}(-\omega)\right]$, where

$$
\begin{gathered}
i \omega \widetilde{\sigma}_{0}(\omega)=2 \Gamma_{\mathrm{L}} \widetilde{\sigma}_{0}(\omega)-\Gamma_{\mathrm{R}}\left[\widetilde{\sigma}_{2 \uparrow} \uparrow(\omega)+\widetilde{\sigma}_{2 \downarrow}(\omega)\right], \quad(37 \mathrm{a}) \\
i \omega \widetilde{\sigma}_{1 \uparrow}(\omega)=-\Gamma_{\mathrm{L}} \widetilde{\sigma}_{0}(\omega)-\Gamma_{\mathrm{R}}\left[\widetilde{\sigma}_{1 \uparrow 2 \uparrow}(\omega)+\widetilde{\sigma}_{1 \uparrow 2 \downarrow}(\omega)\right] \\
-i \Omega\left[\widetilde{\sigma}_{2 \uparrow}^{1 \uparrow}(\omega)-\widetilde{\sigma}_{1 \uparrow}^{2 \uparrow}(\omega)\right]-\rho_{1 \uparrow}^{\mathrm{st}}, \quad(37 \mathrm{~b}) \\
i \omega \widetilde{\sigma}_{2 \uparrow}(\omega)=\left(2 \Gamma_{\mathrm{L}}+\Gamma_{\mathrm{R}}\right) \widetilde{\sigma}_{2 \uparrow}(\omega)+i \Omega\left[\widetilde{\sigma}_{2 \uparrow}^{1 \uparrow}(\omega)-\widetilde{\sigma}_{1 \uparrow}^{2 \uparrow}(\omega)\right]-\rho_{2 \uparrow}^{\mathrm{st}}, \\
i \omega \widetilde{\sigma}_{1 \uparrow 2 \uparrow}(\omega)=\Gamma_{\mathrm{R}} \widetilde{\sigma}_{1 \uparrow 2 \uparrow}(\omega)-\Gamma_{\mathrm{L}} \widetilde{\sigma}_{2 \uparrow}(\omega)-2 \rho_{1 \uparrow 2 \uparrow}^{\mathrm{st}}, \quad(37 \mathrm{~d}) \\
i \omega \widetilde{\sigma}_{2 \uparrow}^{1 \uparrow}(\omega)=-i \Delta \widetilde{\sigma}_{2 \uparrow}^{1 \uparrow}(\omega)-i \Omega\left[\widetilde{\sigma}_{1 \uparrow}(\omega)-\widetilde{\sigma}_{2 \uparrow}(\omega)\right] \\
+\frac{1}{2}\left(2 \Gamma_{\mathrm{L}}+\Gamma_{\mathrm{R}}\right) \widetilde{\sigma}_{2 \uparrow}^{1 \uparrow}(\omega)-\left[\rho^{\mathrm{st}}\right]_{2 \uparrow}^{1 \uparrow} .
\end{gathered}
$$

In resonance $\left(\Delta=E_{2}-E_{1}=0\right)$, we immediately have

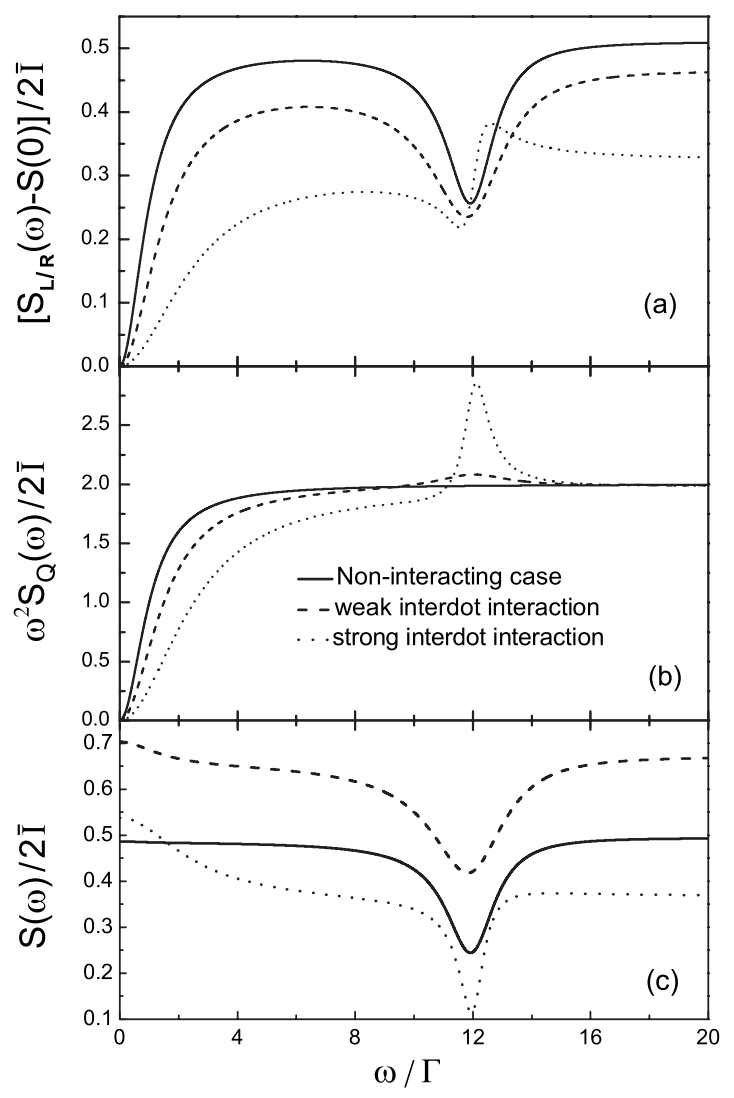

FIG. 3. Noise spectrum of transport current through a pair of coupled quantum dots. Shown in (a) and (b) are the spectral components of the (local) junction currents and the charge-number fluctuations of the double dots, while the result in (c) is their symmetric combination (i.e., $a=b=1 / 2$ ), representing the noise spectrum of the entire circuit current. The solid, dashed, and dotted curves plot, respectively, the results for the noninteracting, single-dot Coulomb blockade, and double-dot Coulomb blockade cases. The adopted parameters are $\Gamma_{\mathrm{L}}=\Gamma_{\mathrm{R}}=\Gamma, \Delta=0$, and $\Omega=6 \Gamma$. The absolute values of the Coulomb interaction energies (i.e., $U_{0}$ and $U_{12}$ ) are irrelevant to the results, which only determine the transport regimes.

$$
\frac{S_{c}(\omega)}{2 \bar{I}}=\frac{\omega^{2}}{2\left[\omega^{2}+\left(\gamma_{1} / 2\right)^{2}\right]} \frac{\gamma_{1}^{4}-6 \Gamma_{\mathrm{L}} \Gamma_{\mathrm{R}} \gamma_{1}^{2}+4\left(\omega^{2}-4 \Omega^{2}\right)^{2}+\gamma_{1}^{2}\left(\omega^{2}+4 \Omega^{2}\right)-8 \Gamma_{\mathrm{L}} \Gamma_{\mathrm{R}}\left(\omega^{2}-4 \Omega^{2}\right)+4\left(4 \Gamma_{\mathrm{L}}^{2}+\Gamma_{\mathrm{R}}^{2}\right) \omega^{2}}{\gamma_{1}^{2} \omega^{2}+\left(\omega^{2}-4 \Omega^{2}\right)^{2}+4 \Gamma_{\mathrm{L}} \Gamma_{\mathrm{R}}\left[\Gamma_{\mathrm{L}} \Gamma_{\mathrm{R}}-\left(\omega^{2}-4 \Omega^{2}\right)\right]} .
$$

Its numerical plot is displayed by the dashed curve in Fig. 3(b).

\section{B. Strong interdot interaction}

This corresponds to the condition $E_{1,2}+U_{12}>\mu_{\mathrm{L}}>\mu_{\mathrm{R}}$ and is referred to as the double-dot Coulomb blockade regime since simultaneous occupation of both dots is forbidden. The involved states are $|0\rangle$ for the two dots empty, $|1 \sigma\rangle$ for the first dot occupied, and $|2 \sigma\rangle$ for the second dot occupied. In this state basis, the master equation reads

$$
\begin{gathered}
\dot{\rho}_{0}=-2 \Gamma_{\mathrm{L}} \rho_{0}+\Gamma_{\mathrm{R}}\left[\rho_{2 \uparrow}+\rho_{2 \downarrow}\right], \\
\dot{\rho}_{1 \uparrow}=i \Omega\left[\rho_{2 \uparrow}^{1 \uparrow}-\rho_{1 \uparrow}^{2 \uparrow}\right]+\Gamma_{\mathrm{L}} \rho_{0}, \\
\dot{\rho}_{2 \uparrow}=-i \Omega\left[\rho_{2 \uparrow}^{1 \uparrow}-\rho_{1 \uparrow}^{2 \uparrow}\right]-\Gamma_{\mathrm{R}} \rho_{2 \uparrow}, \\
\dot{\rho}_{2 \uparrow}^{1 \uparrow}=i \Delta \rho_{2 \uparrow}^{1 \uparrow}+i \Omega\left[\rho_{1 \uparrow}-\rho_{2 \uparrow}\right]-\frac{1}{2} \Gamma_{\mathrm{R}} \rho_{2 \uparrow}^{1 \uparrow} .
\end{gathered}
$$


The same equations for the spin-down components can be derived, and according to Eq. (6) the steady-state current can be easily carried out as

$$
\bar{I}=\frac{\Gamma_{\mathrm{R}} \Omega^{2}}{\Omega^{2}\left(2+\Gamma_{\mathrm{R}} / 2 \Gamma_{\mathrm{L}}\right)+\Gamma_{\mathrm{R}}^{2} / 4+\Delta^{2}} .
$$

In comparison with the previous results ${ }^{45,46}$ a minor difference arises here because of the factor of 2 in front of $\Gamma_{L}$. This factor originates from the spin degree of freedom, which was ignored in the previous work.

To calculate the noise spectrum we use the MacDonald's formula, Eq. (10), again and obtain

$$
S_{\mathrm{L}}(\omega)=-8 \omega \Gamma_{\mathrm{L}} \operatorname{Im}\left[\tilde{N}_{0}(\omega)\right]+4 \Gamma_{\mathrm{L}} \rho_{0}^{\mathrm{st}}-8 \pi \bar{I} \delta(\omega),
$$

where $\rho_{0}^{\text {st }}$ is the stationary solution of Eq. (38) and $\tilde{N}_{0}(\omega)$ satisfies [denoting that $\tilde{\rho}_{0}(\omega)=i \rho_{0}^{\text {st }} /(\omega+i \varepsilon)$ ]

$$
\begin{aligned}
(i \omega-\varepsilon) \tilde{N}_{0}(\omega)= & 2 \Gamma_{\mathrm{L}} \tilde{N}_{0}(\omega)-\Gamma_{\mathrm{R}}\left[\tilde{N}_{2 \uparrow}(\omega)+\tilde{N}_{2 \downarrow}(\omega)\right], \\
(i \omega-\varepsilon) \tilde{N}_{1 \uparrow}(\omega)= & -\Gamma_{\mathrm{L}} \tilde{N}_{0}(\omega)-i \Omega\left[\tilde{N}_{2 \uparrow}^{1 \uparrow}(\omega)-\tilde{N}_{1 \uparrow}^{2 \uparrow}(\omega)\right] \\
& +\Gamma_{\mathrm{L}} \tilde{\rho}_{0}(\omega), \\
(i \omega-\varepsilon) \tilde{N}_{2 \uparrow}(\omega)= & \Gamma_{\mathrm{R}} \tilde{N}_{2 \uparrow}(\omega)+i \Omega\left[\tilde{N}_{2 \uparrow}^{1 \uparrow}(\omega)-\tilde{N}_{1 \uparrow}^{2 \uparrow}(\omega)\right], \\
(i \omega-\varepsilon) \tilde{N}_{2 \uparrow}^{1 \uparrow}(\omega)= & -i \Delta \tilde{N}_{2 \uparrow}^{1 \uparrow}(\omega)-i \Omega\left[\tilde{N}_{1 \uparrow}(\omega)-\tilde{N}_{2 \uparrow}(\omega)\right] \\
& +\frac{1}{2} \Gamma_{\mathrm{R}} \tilde{N}_{2 \uparrow}^{1 \uparrow}(\omega) .
\end{aligned}
$$

The solution of the above equations yields the zerofrequency Fano factor

$$
\begin{aligned}
F_{0}= & S(0) / 2 \bar{I}=1 \\
& -\frac{4 \Gamma_{\mathrm{L}} \Omega^{2}\left[\Gamma_{\mathrm{R}}\left(\Gamma_{\mathrm{R}}^{2}+6 \Gamma_{\mathrm{L}} \Gamma_{\mathrm{R}}+8 \Omega^{2}\right)+4 \Delta^{2}\left(\Gamma_{\mathrm{R}}-2 \Gamma_{\mathrm{L}}\right)\right]}{\left[2 \gamma_{2} \Omega^{2}+\Gamma_{\mathrm{L}}\left(\Gamma_{\mathrm{R}}^{2}+4 \Delta^{2}\right)\right]^{2}},
\end{aligned}
$$

where $\gamma_{2}=4 \Gamma_{L}+\Gamma_{R}$. Again, this result slightly differs from the one in Ref. 46, where the spin degree of freedom is neglected. Following precisely the same procedure, an identical result for $S_{\mathrm{R}}(\omega)$ can be obtained. The frequency dependence of $S_{\mathrm{L} / \mathrm{R}}(\omega)$ is numerically plotted as the dotted line in Fig. 3(a).

The spectrum of charge-number fluctuations on the double dots in the above specified regime reads $S_{c}(\omega)$ $=4 \omega^{2} \operatorname{Re}\left[\widetilde{\sigma}_{1 \uparrow}(\omega)+\widetilde{\sigma}_{2 \uparrow}(\omega)+\widetilde{\sigma}_{1 \uparrow}(-\omega)+\widetilde{\sigma}_{2 \uparrow}(-\omega)\right]$, in which

$$
i \omega \widetilde{\sigma}_{0}(\omega)=2 \Gamma_{\mathrm{L}} \widetilde{\sigma}_{0}(\omega)-\Gamma_{\mathrm{R}}\left[\widetilde{\sigma}_{2 \uparrow}(\omega)+\widetilde{\sigma}_{2 \downarrow}(\omega)\right],
$$

$$
\begin{gathered}
i \omega \widetilde{\sigma}_{1 \uparrow}(\omega)=-\Gamma_{\mathrm{L}} \widetilde{\sigma}_{0}(\omega)-i \Omega\left[\widetilde{\sigma}_{2 \uparrow}^{1 \uparrow}(\omega)-\widetilde{\sigma}_{1 \uparrow}^{2 \uparrow}(\omega)\right]-\rho_{1 \uparrow}^{\mathrm{st}}, \\
i \omega \widetilde{\sigma}_{2 \uparrow}(\omega)=\Gamma_{\mathrm{R}} \widetilde{\sigma}_{2 \uparrow}(\omega)+i \Omega\left[\widetilde{\sigma}_{2 \uparrow}^{1 \uparrow}(\omega)-\widetilde{\sigma}_{1 \uparrow}^{2 \uparrow}(\omega)\right]-\rho_{2 \uparrow}^{\mathrm{st}}, \\
i \omega \widetilde{\sigma}_{2 \uparrow}^{1 \uparrow}(\omega)=-i \Delta \widetilde{\sigma}_{2 \uparrow}^{1 \uparrow}(\omega)-i \Omega\left[\widetilde{\sigma}_{1 \uparrow}(\omega)-\widetilde{\sigma}_{2 \uparrow}(\omega)\right]+\frac{1}{2} \Gamma_{\mathrm{R}} \widetilde{\sigma}_{2 \uparrow}^{1 \uparrow}(\omega) \\
-\left[\rho^{\mathrm{st}}\right]_{2 \uparrow}^{1 \uparrow},
\end{gathered}
$$

with $\rho_{1 \uparrow}^{\text {st }}, \rho_{2 \uparrow}^{\text {st }}$, and $\left[\rho^{\text {st }}\right]_{2 \uparrow}^{1 \uparrow}$ being stationary solutions of Eq. (38). In resonance $(\Delta=0)$ we have [see the numerical result, the dotted line in Fig. 3(b)]

$$
\frac{S_{c}(\omega)}{2 \bar{I}}=\frac{2 \omega^{2}\left[4\left(\omega^{2}-4 \Omega^{2}\right)^{2}+\Gamma_{\mathrm{R}}^{2}\left(\omega^{2}+4 \Omega^{2}\right)+\Gamma_{\mathrm{R}}^{2}\left(\Gamma_{\mathrm{R}}^{2}+4 \omega^{2}\right)\right]}{\left(\gamma_{2}^{2}+4 \omega^{2}\right)\left(\omega^{2}-4 \Omega^{2}\right)^{2}-8 \Gamma_{\mathrm{L}} \Gamma_{\mathrm{R}} \omega^{2}\left(\omega^{2}-4 \Omega^{2}\right)+16 \Gamma_{\mathrm{L}} \Gamma_{\mathrm{R}}^{2} \gamma_{2} \Omega^{2}+\Gamma_{\mathrm{R}}^{2}\left(\omega^{2}+4 \Gamma_{\mathrm{L}}^{2}\right)\left(\Gamma_{\mathrm{R}}^{2}+4 \omega^{2}\right)+4 \Gamma_{\mathrm{L}}^{2} \Gamma_{\mathrm{R}}^{2} \omega^{2}} .
$$

Now we present brief discussions of the numerical results in Fig. 3. For comparative purposes, the result for noninteracting double dots, which corresponds to the voltage regime $\mu_{\mathrm{L}}>E_{1,2}+U_{0}\left(U_{12}\right)>\mu_{\mathrm{R}}$, is also included by the solid curves. (i) For electron transport of two coherently coupled quantum dots, the transport electron will experience coherent oscillations between the two dots. This effect is much stronger when coherent coupling dominates at large $\Omega$. In this case, once an electron is injected from the emitter to the first dot, it will periodically oscillate between the two dots until it is finally tunneled out from the second dot to the collector, which happens rarely due to weak tunnel coupling. The periodic oscillation of the electron between the two dots strongly suppresses another electron from entering the dot. It is this effect that leads to dips of the circuit current noise spectrum occurring near the coherent oscillation frequency $\omega=2 \Omega$ when in resonance $(\Delta=0)$; see Fig. 3(c). (ii) The interplay between the Coulomb interaction and the coherent oscillation also affects the current spectrum. Impressively, for $S_{\mathrm{L} / \mathrm{R}}(\omega)$, the dip at the frequency $\omega=2 \Omega$ would evolve to an inflection, as the Coulomb interaction makes the system in the double-dot Coulomb blockade regime; i.e., doubleelectron occupation of the coupled dots is forbidden. (iii) In the double-dot Coulomb blockade regime, the chargenumber fluctuation spectrum shown in Fig. 3(b) by the dotted curve always has a peak at $\omega=2 \Omega$, owing to the coherent 
oscillation as mentioned above. On the other hand, in the noninteracting and single-dot Coulomb blockade regimes, whether the charge-number fluctuation spectrum has a peak at $\omega=2 \Omega$ or not depends on the geometry of the transport setup. For instance, the solid curve in Fig. 3(b) for the noninteracting system does not show a peak in the symmetric setup (i.e., $\Gamma_{L}=\Gamma_{R}$ ), whereas a peak at $\omega=2 \Omega$ can appear in the asymmetric case-e.g., $\Gamma_{\mathrm{L}}=5 \Gamma_{\mathrm{R}}$ as we have checked.

In concluding this section, let us briefly comment on some related work on the frequency-dependent noise through coupled double-dot systems. The fact that noise spectrum can provide detailed information on the internal and dissipative dynamics has been demonstrated by Aguado and Brandes in a single-electron two-level system coupled to a boson bath. ${ }^{42}$ It has also been demonstrated by Djuric et al. in a similar system, but with Coulomb charging energy included in the absence of bosonic dissipation. ${ }^{47}$ The present double-dot structure is similar to that in Ref. 47, but with both quantum dot levels tunneling coupled to the leads. It has in fact been proposed as an alternative detector of a charge qubit. ${ }^{48}$

\section{CONCLUSION AND DISCUSSION}

To summarize, based on the $n$-resolved quantum master equation we have presented an efficient method of calculating the current noise spectrum. The major approximation involved in the our master equation approach is the secondorder Born approximation for the tunnel Hamiltonian. This approximation sets restriction on valid temperature regime ${ }^{40}$-i.e., $k_{\mathrm{B}} T \gg \Gamma$. Fortunately, in most cases the system coupling to the electrodes is indeed very weak; then, the present formalism remains appropriate for a rather wide range of temperature. Also, the excellent agreement with other approaches, as shown by previous work $^{33-36}$ and the present one, clearly indicates its applicability to quantum transport. For temperatures indeed lower than the tunneling width, exact hierarchical equations of motion have recently been established by us on the basis of the calculus-on-pathintegral algorithm. ${ }^{49}$ Also, higher-order tunneling contributions due to low temperatures, in principal, can be included by generating to higher tiers in terms of the tunnel-coupling strength.
Another assumption is the wideband approximation that we have used in the application of our approach. It yields energy-independent rates, since the Fermi functions can be well approximated by either one or zero at large bias. Moreover, the imaginary part of the rate, which accounts for the energy level renormalization, ${ }^{13,40}$ is also neglected at large bias voltage in our application. However, the inclusion of this energy renormalization can be carried out readily within our present second-order theory. As a matter of fact, it has already been done in the context of quantum dissipation dynamics; see, e.g., Refs. 39 and 50. In the present application, for the reason of the construction of analytical results for some simple systems, these effects are neglected under the condition of large bias limit.

The present theory can be considered as a finitetemperature extension of the $n$-resolved quantum Bloch-type equation proposed by Gurvitz and Prager. ${ }^{28}$ From the theoretical construction point of view, both involve the decomposition of reservoirs coupling to individual $n$-electron tunneling events. However, their approach is based on a wavefunction construction; thus, the effect of finite temperature remains as a challenge. The present approach based on the reduced density matrix has the advantage in its simplicity of treating properly a broad range of dissipation, in relation to measurement signals, including the full counting statistics. $^{51-53}$ In comparison with the Landauer-Büttiker scattering matrix approach, ${ }^{14}$ the present $n$-resolved reduced density matrix theory has the advantage of its transparency in the involving dynamics and many-body interaction. The above advantages have now been demonstrated in the present work by considering quantum transport through some simple quantum-dot systems in the presence of Coulomb interaction and quantum coherence.

\section{ACKNOWLEDGMENTS}

Support from the National Natural Science Foundation of China under Grants No. 60376037, No. 60425412, and No. 90503013, the Major State Basic Research Project No. G001CB3095 of China, and the Research Grants Council of the Hong Kong Government (Grant No. 605105) are gratefully acknowledged. *xqli@red.semi.ac.cn

${ }^{1}$ E. V. Sukhorukov, G. Burkard, and D. Loss, Phys. Rev. B 63, 125315 (2001).

${ }^{2}$ G. Kießlich, A. Wacker, and E. Schöll, Phys. Rev. B 68, 125320 (2003).

${ }^{3}$ A. Thielmann, M. H. Hettler, J. König, and G. Schön, Phys. Rev. B 71, 045341 (2005).

${ }^{4}$ A. Thielmann, M. H. Hettler, J. König, and G. Schön, Phys. Rev. Lett. 95, 146806 (2005).

${ }^{5}$ J. Aghassi, A. Thielmann, M. H. Hettler, and G. Schön, Appl. Phys. Lett. 89, 052101 (2006).

${ }^{6}$ A. Cottet, W. Belzig, and C. Bruder, Phys. Rev. Lett. 92, 206801
(2004).

${ }^{7}$ H. Birk, M. J. M. de Jong, and C. Schönenberger, Phys. Rev. Lett. 75, 1610 (1995).

${ }^{8}$ H. C. Liu, J. Li, G. C. Aers, C. R. Leavens, M. Buchanan, and Z. R. Wasilewski, Phys. Rev. B 51, 5116 (1995).

${ }^{9}$ A. Przadka, K. J. Webb, D. B. Janes, H. C. Liu, and Z. R. Wasilewski, Appl. Phys. Lett. 71, 530 (1997).

${ }^{10}$ S. S. Safonov, A. K. Savchenko, D. A. Bagrets, O. N. Jouravlev, Y. V. Nazarov, E. H. Linfield, and D. A. Ritchie, Phys. Rev. Lett. 91, 136801 (2003).

${ }^{11}$ A. Nauen, F. Hohls, N. Maire, K. Pierz, and R. J. Haug, Phys. Rev. B 70, 033305 (2004). 
${ }^{12}$ S. W. Jung, T. Fujisawa, Y. Hirayama, and Y. H. Jeong, Appl. Phys. Lett. 85, 768 (2004).

${ }^{13}$ M. Braun, J. König, and J. Martinek, arXiv:cond-mat/0601366 (unpublished).

${ }^{14}$ Y. M. Blanter and M. Büttiker, Phys. Rep. 336, 1 (2000).

${ }^{15}$ M. Büttiker, Physica B 175, 199 (1991).

${ }^{16}$ L. Y. Chen and C. S. Ting, Phys. Rev. B 43, 4534 (1991).

${ }^{17}$ K.-M. Hung and G. Y. Wu, Phys. Rev. B 48, 14687 (1993).

${ }^{18}$ A. Thielmann, M. H. Hettler, J. König, and G. Schön, Phys. Rev. B 68, 115105 (2003).

${ }^{19}$ H. Schoeller and G. Schön, Phys. Rev. B 50, 18436 (1994).

${ }^{20}$ M. J. M. de Jong and C. W. J. Beenakker, Phys. Rev. B 51, 16867 (1995)

${ }^{21}$ M. J. M. de Jong and C. W. J. Beenakker, Physica A 230, 219 (1996).

${ }^{22}$ J. H. Davies, P. Hyldgaard, S. Hershfield, and J. W. Wilkins, Phys. Rev. B 46, 9620 (1992).

${ }^{23}$ J. C. Egues, S. Hershfield, and J. W. Wilkins, Phys. Rev. B 49, 13517 (1994).

${ }^{24}$ A. N. Korotkov, Phys. Rev. B 49, 10381 (1994).

${ }^{25}$ S. Hershfield, J. H. Davies, P. Hyldgaard, C. J. Stanton, and J. W. Wilkins, Phys. Rev. B 47, 1967 (1993).

${ }^{26}$ U. Hanke, Y. M. Galperin, K. A. Chao, and N. Zou, Phys. Rev. B 48, 17209 (1993).

${ }^{27}$ H. B. Sun and G. J. Milburn, Phys. Rev. B 59, 10748 (1999).

${ }^{28}$ S. A. Gurvitz and Y. S. Prager, Phys. Rev. B 53, 15932 (1996).

${ }^{29}$ D. Mozyrsky, L. Fedichkin, S. A. Gurvitz, and G. P. Berman, Phys. Rev. B 66, 161313(R) (2002).

${ }^{30}$ S. A. Gurvitz, IEEE Trans. Nanotechnol. 4, 45 (2005).

${ }^{31}$ C. Flindt, T. Novotný, and A.-P. Jauho, Phys. Rev. B 70, 205334 (2004).

${ }^{32}$ T. Novotný, A. Donarini, C. Flindt, and A.-P. Jauho, Phys. Rev. Lett. 92, 248302 (2004).

${ }^{33}$ P. Cui, X. Q. Li, J. S. Shao, and Y. J. Yan, Phys. Lett. A 357, 449 (2006).
${ }^{34}$ X. Q. Li, W. K. Zhang, P. Cui, J. S. Shao, Z. S. Ma, and Y. J. Yan, Phys. Rev. B 69, 085315 (2004).

${ }^{35}$ X. Q. Li, P. Cui, and Y. J. Yan, Phys. Rev. Lett. 94, 066803 (2005).

${ }^{36}$ X. Q. Li, J. Y. Luo, Y. G. Yang, P. Cui, and Y. J. Yan, Phys. Rev. B 71, 205304 (2005).

${ }^{37}$ Y. J. Yan, Phys. Rev. A 58, 2721 (1998).

${ }^{38}$ Since the transport circuit is closed, extra electrons arrived at the collector will flow back into the emitter via the external circuit. This rapid relaxation processes quickly bring the reservoirs to the local thermal equilibrium state.

${ }^{39}$ Y. J. Yan and R. X. Xu, Annu. Rev. Phys. Chem. 56, 187 (2005).

${ }^{40}$ B. Wunsch, M. Braun, J. König, and D. Pfannkuche, Phys. Rev. B 72, 205319 (2005).

${ }^{41}$ S. A. Gurvitz, D. Mozyrsky, and G. P. Berman, Phys. Rev. B 72, 205341 (2005).

${ }^{42}$ R. Aguado and T. Brandes, Phys. Rev. Lett. 92, 206601 (2004).

${ }^{43}$ D. K. C. MacDonald, Noise and Fluctuations: An Introduction (Wiley, New York, 1962), Chap. 2.2.1.

${ }^{44}$ G. Kießlich, P. Samuelsson, A. Wacker, and E. Schöll, Phys. Rev. B 73, 033312 (2006).

${ }^{45}$ T. H. Stoof and Y. V. Nazarov, Phys. Rev. B 53, 1050 (1996).

${ }^{46}$ B. Elattari and S. A. Gurvitz, Phys. Lett. A 292, 289 (2002).

${ }^{47}$ I. Djuric, B. Dong, and H. L. Cui, Appl. Phys. Lett. 87, 032105 (2005).

${ }^{48}$ T. Gilad and S. A. Gurvitz, Phys. Rev. Lett. 97, 116806 (2006).

${ }^{49}$ J. S. Jin, S. Welack, J. Y. Luo, X. Q. Li, P. Cui, R. X. Xu, and Y. J. Yan, J. Chem. Phys. 126, 134113 (2007).

${ }^{50}$ R. X. Xu and Y. J. Yan, J. Chem. Phys. 116, 9196 (2002).

${ }^{51}$ D. A. Bagrets and Y. V. Nazarov, Phys. Rev. B 67, 085316 (2003).

${ }^{52}$ S.-K. Wang, H. Jiao, F. Li, X.-Q. Li, and Y. J. Yan, arXiv:condmat/0703745 (unpublished).

${ }^{53}$ L. S. Levitov, H. W. Lee, and G. B. Lesovik, J. Math. Phys. 37, 4845 (1996). 